\title{
RADIO FREQUENCY ABLATION OF RENAL CELL CARCINOMA VIA IMAGE GUIDED NEEDLE ELECTRODES
}

\author{
FRANCIS J. McGOVERN, BRADFORD J. WOOD, S. NAHUM GOLDBERG AND PETER R. MUELLER \\ From the Departments of Urology and Radiology, Massachusetts General Hospital and Harvard Medical School, Boston, Massachusetts, \\ and Department of Radiology, National Institutes of Health, Bethesda, Maryland
}

KEY WoRDS: kidney; carcinoma, renal cell; neoplasms; ultrasonography

Radio frequency ablation of small focal neoplasms has previously been demonstrated in other organs, such as the liver and bone.1,2 To our knowledge we report the first completely treated case of radio frequency ablation of renal cell carcinoma via percutaneous placement of a needle electrode under ultrasound guidance.

\section{CASE REPORT}

An 84-year-old man was diagnosed with a solid renal mass and he refused open surgery. Computerized tomography (CT) demonstrated mass enhancement with iodinated contrast material, consistent with solid renal cell carcinoma (fig. 1). Three years later the mass had enlarged to $3.5 \mathrm{~cm}$. The patient continued to refuse surgery but he consented to percutaneous radio frequency ablation. Institutional review board permission was obtained before the procedure.

The patient underwent conscious sedation with $2 \mathrm{mg}$. midazolam and $150 \mathrm{mg}$. fentanyl given intravenously as well as $1 \%$ lidocaine given subcutaneously. Immediately before ablation percutaneous needle biopsy confirmed the diagnosis of renal cell carcinoma. A 17 gauge, internally cooled radio frequency needle electrode was placed percutaneously under real-time ultrasound guidance into the center of the lesion. Goldberg $^{1}$ and Solbiati $^{2}$ et al have shown that these electrodes induce large volumes of coagulation necrosis in liver tumors. The tumor was heated to $90 \mathrm{C}$ for 1 minute, followed by deposition of 1,200 mA. for 12 minutes. Ultrasonography revealed progressive hyperechogenicity enveloping the lesion during treatment. After ablation temperatures remained at greater than $60 \mathrm{C}$ for 3.5 minutes. The electrode was removed without incident.

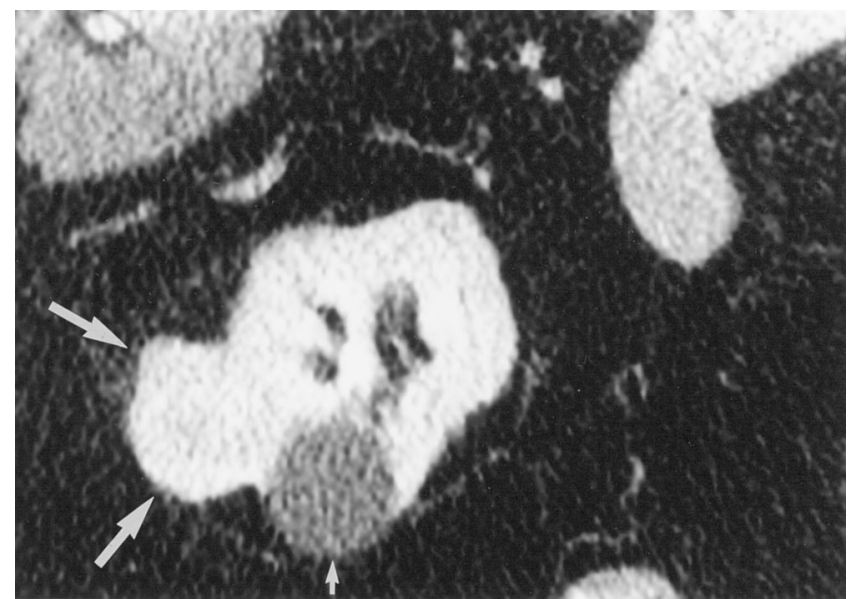

FIG. 1. Preoperatively enhanced CT demonstrates marked enhancement of solid exophytic tumor (large arrows). Incidental simple cyst abuts tumor (small arrow).
The patient was awake, comfortable and conversant, and vital signs were stable throughout the procedure as well as during 2 hours of postoperative monitoring. CT 2 hours postoperatively revealed a $3.5 \mathrm{~cm}$. nonenhancing region enveloping the previously enhancing tumor, and no evidence of complications, such as bleeding or urinoma (fig. 2).

The patient was discharged home in stable condition. $\mathrm{He}$ was followed closely during the following month, and he reported no flank pain or hematuria. Followup imaging at 1 and 3 months showed persistent nonenhancement of the treated region with no evidence of peripheral enhancement suggestive of residual viable tumor.

\section{DISCUSSION}

With the advent of abdominal CT and magnetic resonance imaging the detection of small asymptomatic renal tumors has increased. While the standard of care dictates radical or partial nephrectomy, many patients with this incidental finding are poor surgical candidates due to advanced age or co-morbid disease. We present a new minimally invasive treatment alternative for these lesions involving radio frequency ablation using a needle electrode placed percutaneously under ultrasound guidance. Zlotta et al reported that this radio frequency technique is safe ex vivo and in vivo perioperatively. ${ }^{3}$ It may be performed on an outpatient basis using conscious sedation. Postoperatively imaging findings are similar to those reported by Goldberg ${ }^{1}$ and Solbiati $^{2}$ et al for liver tumor ablation.

Fat surrounding a kidney lesion may provide an insulating effect that results in greater heat retention and, thus, more tumor cell death compared to the liver. ${ }^{1,2}$ Radio frequency ablation warrants additional future study with extended followup to determine long-term efficacy. In conclusion, successful percutaneous ablation may prove to be a reasonable

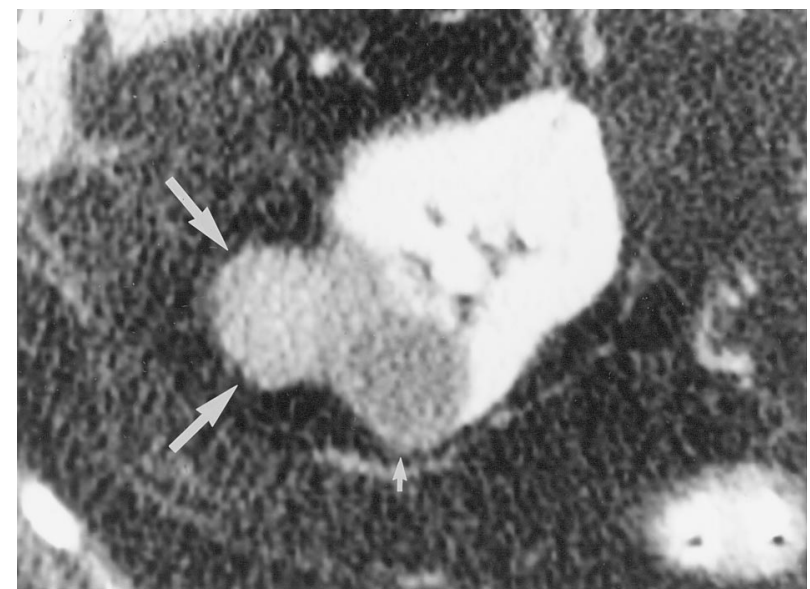

FIG. 2. Postoperatively enhanced CT shows complete obliteration of tumor identified by zero contrast medium enhancement (large arrows). Simple cyst immediately adjacent to treated area is unchanged (small arrow). 
treatment alternative in patients who are at significant operative risk or when renal preservation is desired, such as those in whom von-Hippel Lindau disease or other conditions predispose to multiple bilateral renal tumors.

\section{REFERENCES}

1. Goldberg, S. N., Livraghi, T., Solbiati, L. and Gazelle, G. S.: In situ ablation of focal hepatic neoplasms. In: Hepatobiliary and Pancreatic Radiology: Imaging and Intervention. Edited by
G. S. Gazelle, S. Saini and P. R. Mueller. New York: Thieme Medical Pub., 1997.

2. Solbiati, L., Goldberg, S. N., Ierace, T., Livraghi, T., Sironi, S. and Gazelle, G. S.: Radiofrequency ablation of hepatic metastases with cooled-tip electrodes: results in 29 patients. Radiology, 205: 367, 1997.

3. Zlotta, A. R., Wildschultz, T., Raviv, G., Peny, M. O., vanGansbeke, D., Noel, J. and Schulman, C. C.: Radiofrequency interstitial tumor ablation (RITA) is a possible new modality for treatment of renal cancer: ex vivo and in vivo experience. J. Endourol., 11: 251, 1997. 